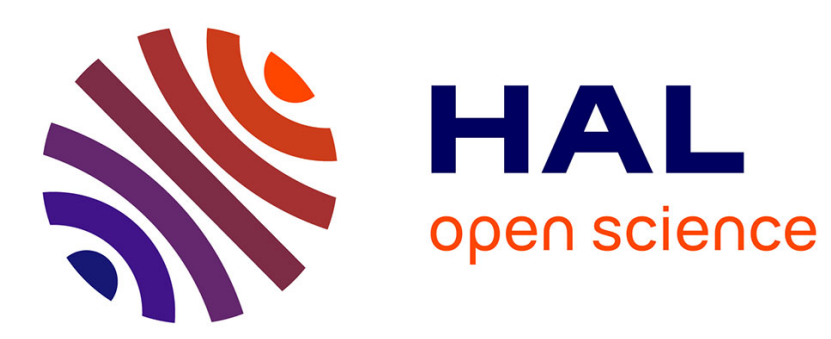

\title{
Global existence for a strongly coupled reaction-diffusion systems with nonlinearities of exponential growth
}

Belgacem Rebiai, Saïd Benachour

\section{To cite this version:}

Belgacem Rebiai, Saïd Benachour. Global existence for a strongly coupled reaction-diffusion systems with nonlinearities of exponential growth. 2010. hal-00454730

\author{
HAL Id: hal-00454730 \\ https://hal.science/hal-00454730
}

Preprint submitted on 9 Feb 2010

HAL is a multi-disciplinary open access archive for the deposit and dissemination of scientific research documents, whether they are published or not. The documents may come from teaching and research institutions in France or abroad, or from public or private research centers.
L'archive ouverte pluridisciplinaire HAL, est destinée au dépôt et à la diffusion de documents scientifiques de niveau recherche, publiés ou non, émanant des établissements d'enseignement et de recherche français ou étrangers, des laboratoires publics ou privés. 


\title{
GLOBAL EXISTENCE FOR A STRONGLY COUPLED REACTION-DIFFUSION SYSTEMS WITH NONLINEARITIES OF EXPONENTIAL GROWTH
}

\author{
BELGACEM REBIAI AND SAÏD BENACHOUR
}

\begin{abstract}
The aim of this study is to construct the invariant regions in which we can establish the global existence of classical solutions for reaction-diffusion systems with a general full matrix of diffusion coefficients. Our techniques are based on invariant regions and Lyapunov functional methods. The nonlinear reaction term has been supposed to be of exponential growth.
\end{abstract}

\section{Introduction}

In this work, we are interested in global existence of classical solutions to the following reaction-diffusion system

$$
\begin{aligned}
& \frac{\partial u}{\partial t}-a_{11} \Delta u-a_{12} \Delta v=f(u, v) \quad \text { in } \quad(0,+\infty) \times \Omega \\
& \frac{\partial v}{\partial t}-a_{21} \Delta u-a_{22} \Delta v=g(u, v) \quad \text { in } \quad(0,+\infty) \times \Omega,
\end{aligned}
$$

with the initial conditions:

$$
u(0, x)=u_{0}(x), \quad v(0, x)=v_{0}(x) \quad \text { in } \quad \Omega,
$$

and the homogeneous boundary conditions:

$$
\alpha u+(1-\alpha) \frac{\partial u}{\partial \nu}=0, \quad \alpha v+(1-\alpha) \frac{\partial v}{\partial \nu}=0 \text { on }(0,+\infty) \times \partial \Omega,
$$

where $\Omega$ is an open bounded domain of class $C^{1}$ in $\mathbb{R}^{n}, \frac{\partial}{\partial \nu}$ denotes the outward normal derivative on $\partial \Omega, \alpha$ is a function of class $C^{1}$ on $\partial \Omega$ such that $0 \leq \alpha \leq 1$ and the diffusion terms $a_{i j}, i, j=1,2$ are supposed to be positive constants such that

$$
\left(a_{12}+a_{21}\right)^{2}<4 a_{11} a_{22},
$$

Date: January 20, 2010.

2000 Mathematics Subject Classification. 35K45, 35K57.

Key words and phrases. Reaction diffusion systems, invariant regions, Lyapunov functionals, global existence. 
which reflects the parabolicity of the system and implies at the same time that the matrix of diffusion

$$
A=\left(\begin{array}{ll}
a_{11} & a_{12} \\
a_{21} & a_{22}
\end{array}\right)
$$

is positive definite. The eigenvalues $\lambda_{1}$ and $\lambda_{2}\left(\lambda_{1}<\lambda_{2}\right)$ of $A$ are positive.

If we put

$$
\underline{a}=\min \left\{a_{11}, a_{22}\right\} \quad \text { and } \quad \bar{a}=\max \left\{a_{11}, a_{22}\right\}
$$

then, the positivity of the diffusion terms implies that

$$
\lambda_{1}<\underline{a} \leq \bar{a}<\lambda_{2} .
$$

We also put

$$
\begin{aligned}
& \Sigma_{1}=\left\{(r, s) \in \mathbb{R}^{2}: \quad \mu_{2} r \leq s \leq \mu_{1} r\right\} \\
& \Sigma_{2}=\left\{(r, s) \in \mathbb{R}^{2}: \quad \frac{1}{\mu_{2}} s \leq r \leq \frac{1}{\mu_{1}} s\right\},
\end{aligned}
$$

where

$$
\mu_{1}=\frac{a_{21}}{a_{11}-\lambda_{1}}>0>\mu_{2}=\frac{a_{21}}{a_{11}-\lambda_{2}}
$$

We suppose:

(A1) $\quad f$ and $g$ are continuously differentiable on $\Sigma_{1} \cup \Sigma_{2}$,

$$
\begin{aligned}
& (-1)^{j}(f(r, s), g(r, s)) \in \Sigma_{j} \quad \text { and } \mu_{i} f\left(r, \mu_{i} r\right)=g\left(r, \mu_{i} r\right) \\
& \text { for all }(r, s) \in \Sigma_{i}, i, j=1,2(j \neq i), \\
& g(r, s)-\mu_{j} f(r, s) \leq(-1)^{j} \psi\left(s-\mu_{j} r\right)\left(g(r, s)-\mu_{i} f(r, s)\right) \\
& \text { for all }(r, s) \in \Sigma_{i}, i, j=1,2(j \neq i),
\end{aligned}
$$

where $\psi$ is a nonnegative continuously differentiable function on $[0,+\infty)$ such that there exists a constant $\beta \geq 1$ satisfying $\lim _{\eta \rightarrow+\infty} \eta^{\beta-1} \psi(\eta)=\ell$ where $\ell$ is a nonnegative constant,

$$
\begin{aligned}
& g(r, s)-\mu_{j} f(r, s) \leq C \varphi\left((-1)^{i}\left(s-\mu_{i} r\right)\right) e^{\alpha\left(s-\mu_{j} r\right)^{\beta}} \\
& \text { for all }(r, s) \in \Sigma_{i}, i, j=1,2(j \neq i),
\end{aligned}
$$

where $C>0, \alpha>0, \beta$ is the same as in (A3) and $\varphi$ is any nonnegative continuously differentiable function on $[0,+\infty)$ such that $\varphi(0)=0$.

The initial data are assumed to be in $\Sigma$ where $\Sigma=\Sigma_{1}$ or $\Sigma=\Sigma_{2}$. 
The present investigation is a continuation of results obtained in [24]. In this study, we will treat the case of a general full matrix of diffusion coefficients and prove that if $f$ and $g$ satisfying (A1)-(A4), then $\Sigma$ is an invariant region for problem (1.1)-(1.4). Once the invariant regions are constructed, we demonstrate that for any initial data in $\Sigma$ satisfying

$$
\left\|\mu_{i} u_{0}-v_{0}\right\|_{\infty}<\frac{8 \lambda_{1} \lambda_{2}}{\alpha \beta \ell n\left(\lambda_{1}-\lambda_{2}\right)^{2}}, \ell>0 \text { when } \Sigma=\Sigma_{i}, i=1,2,
$$

problem (1.1)-(1.4) is equivalent to a problem for which the global existence follows from the technique based on Lyapunov functional method (see, e.g., [3], [8], [14], [16], [18], [21] and [24]).

In [12] J. I. Kanel and M. Kirane proved the global existence of solutions for a strongly coupled reaction-diffusion system with homogeneous Neumann boundary conditions and

$$
g(u, v)=-f(u, v)=u v^{m}, \quad m>0 \text { is an odd integer, }
$$

under the conditions

$$
\begin{array}{ll}
\text { - } & 0<a_{22}-a_{11}<a_{21}, \\
\text { - } & 0<a_{12} \ll 1, \\
\text { - } & \left|a_{22}-a_{11}+a_{12}-a_{21}\right|<\frac{\gamma_{1}+1}{\gamma_{1} C_{p}},
\end{array}
$$

where

$$
\gamma_{1}=\frac{a_{22}-a_{11}-\sqrt{\left(a_{22}-a_{11}\right)^{2}+4 a_{12} a_{21}}}{2 a_{12}}<-1
$$

and $C_{p}$ is the same constant used in Theorem 1 of [20]. Later they improved their results in [13] where they obtained the global existence under the following assumptions

- $a_{22}<a_{11}+a_{21}$,

- $a_{12}<\varepsilon_{0}=\frac{a_{11} a_{22}\left(a_{11}+a_{21}-a_{22}\right)}{a_{11} a_{22}+a_{21}\left(a_{11}+a_{21}-a_{22}\right)}$ if $a_{11} \leq a_{22}<a_{11}+a_{21}$,

- $a_{12}<\min \left\{\frac{1}{2}\left(a_{11}+a_{21}\right), \varepsilon_{0}\right\} \quad$ if $a_{22}<a_{11}$,

and

- $|F(v)| \leq C_{F}\left(1+|v|^{1-\varepsilon}\right), \quad v F(v) \geq 0 \quad$ for all $\quad v \in \mathbb{R}$,

where $C_{F}>0, \varepsilon$ is any constant such that $0<\varepsilon<1$ and

$$
g(u, v)=-f(u, v)=u F(v) .
$$


On the same direction, S. Kouachi [17] has proved the global existence of solutions for two-component reaction-diffusion systems with a general full matrix of diffusion coefficients, nonhomogeneous boundary conditions and polynomial growth conditions on the nonlinear terms and he obtained in [16] the global existence of solutions for the same system with homogeneous Neumann boundary conditions and

$$
g(u, v)=\rho F(u, v), \quad f(u, v)=-\sigma F(u, v), \quad \rho>0, \quad \sigma>0,
$$

where

- $F(u, v) \leq C e^{\alpha v^{\beta}}, C>0, \alpha>0,0<\beta \leq 1$, when $-\mu_{2}>\frac{\rho}{\sigma}$,

- $F(u, v) \leq C e^{\alpha u^{\beta}}, C>0, \alpha>0,0<\beta \leq 1$, when $-\mu_{2}<\frac{\rho}{\sigma}$,

under these conditions

- $\left\|u_{0}-\mu_{2} v_{0}\right\|_{\infty}<\frac{-8 \lambda_{1} \lambda_{2} \mu_{1}\left(\rho+\sigma \mu_{2}\right)}{\alpha n \mu_{2}\left(\rho+\sigma \mu_{1}\right)\left(\lambda_{1}-\lambda_{2}\right)^{2}}, \quad$ when $\quad-\mu_{2}>\frac{\rho}{\sigma}$,

- $\left\|u_{0}-\mu_{1} v_{0}\right\|_{\infty}<\frac{8 \lambda_{1} \lambda_{2} \mu_{2}\left(\rho+\sigma \mu_{1}\right)}{\alpha n \mu_{1}\left(\rho+\sigma \mu_{2}\right)\left(\lambda_{1}-\lambda_{2}\right)^{2}}, \quad$ when $-\mu_{2}<\frac{\rho}{\sigma}$,

where $\mu_{1}$ and $\mu_{2}$ are the same as in (1.5).

Many chemical and biological operations are described by reaction diffusion systems with a full matrix of diffusion coefficients. The components $u(t, x)$ and $v(t, x)$ can be represent either chemical concentrations or biological population densities (see, e.g., E. L. Cussler [5] and $[6])$.

We note that the resolution of the problem (1.1)-(1.4) is quite more difficult. As a consequence of the blow-up examples found in [23], we can prove that there is blow-up of the solutions in finite time for such full systems even though the initial data are regular, the solutions are positive and the nonlinear terms are negative, a structure that ensured the global existence in the diagonal case.

Our goal is to understand how the results of the diagonal case extend to the nondiagonal situation without any additional assumption on the diffusion coefficients in the case of possiblity of growth faster than exponential for the reaction terms. For this purpose, we construct the invariant regions in which we can demonstrate that for any initial data in this regions satisfying (1.6), problem (1.1)-(1.4) is equivalent to a problem for which the global existence follows from the same Lyapunov 
functional used in [24] when the reactive terms satisfies (A1)-(A4).

Throughout this work, we denote by $\|\cdot\|_{p}, \quad p \in[1,+\infty)$ the norm in $L^{p}(\Omega)$ and $\|\cdot\|_{\infty}$ the norm in $C(\bar{\Omega})$ or $L^{\infty}(\Omega)$.

\section{Local existence and invariant regions}

The study of local existence and uniqueness of solutions $(u, v)$ of (1.1)-(1.4) follows from the basic existence theory for parabolic semilinear equations (see, e.g., [2], [9], [11] and [22]). As a consequence, for any initial data in $C(\bar{\Omega})$ or $L^{\infty}(\Omega)$ there exists a $T^{*} \in(0,+\infty]$ such that (1.1)-(1.4) has a unique classical solution on $\left[0, T^{*}\right) \times \Omega$. Furthermore, if $T^{*}<+\infty$, then

$$
\lim _{t \uparrow T^{*}}\left(\|u(t)\|_{\infty}+\|v(t)\|_{\infty}\right)=+\infty .
$$

Therefore, if there exists a positive constant $C$ such that

$$
\|u(t)\|_{\infty}+\|v(t)\|_{\infty} \leq C \text { for all } t \in\left[0, T^{*}\right),
$$

then, $T^{*}=+\infty$.

Since the initial conditions are in $\Sigma$, then under the assumptions (A1)-(A2), the next proposition says that the classical solution of (1.1)-(1.4) on $\left[0, T^{*}\right) \times \Omega$ remains in $\Sigma$ for all $t$ in $\left[0, T^{*}\right)$.

Proposition 2.1. Suppose that the assumptions $(\boldsymbol{A 1})$-(A2) are satisfied. Then for any $\left(u_{0}, v_{0}\right)$ in $\Sigma$ the classical solution $(u, v)$ of problem (1.1)-(1.4) on $\left[0, T^{*}\right) \times \Omega$ remains in $\Sigma$ for all $t$ in $\left[0, T^{*}\right)$.

Proof of Proposition 2.1. One starts with the case where $\Sigma=\Sigma_{1}$. Multiplying equations (1.1) one time through by $\mu_{1}$ and subtracting (1.2) and another time by $-\mu_{2}$ and adding (1.2), then if we put

- $z_{1}=\mu_{1} u-v \quad$ and $\quad z_{2}=-\mu_{2} u+v$ for all $(u, v) \in \Sigma_{1}$,

- $F_{1}=-\mu_{1} f+g$ and $F_{2}=-\mu_{2} f+g$ for all $(u, v) \in \Sigma_{1}$,

we get

$$
\begin{aligned}
& \frac{\partial z_{1}}{\partial t}-\lambda_{1} \Delta z_{1}=-F_{1}\left(z_{1}, z_{2}\right) \quad \text { in } \quad(0,+\infty) \times \Omega \\
& \frac{\partial z_{2}}{\partial t}-\lambda_{2} \Delta z_{2}=F_{2}\left(z_{1}, z_{2}\right) \quad \text { in } \quad(0,+\infty) \times \Omega .
\end{aligned}
$$

with the initial conditions:

$$
z_{i}(0, x)=z_{i}^{0}(x), \quad i=1,2, \quad \text { in } \Omega,
$$


and the homogeneous boundary conditions:

$$
\alpha z_{i}+(1-\alpha) \frac{\partial z_{i}}{\partial \nu}=0, \quad i=1,2, \quad \text { on } \quad(0,+\infty) \times \partial \Omega,
$$

Since $\lambda_{1}$ and $\lambda_{2}$ are the eigenvalues of the matrix $A^{t},(1.1)-(1.4)$ is equivalent to (2.1)-(2.4). Then to prove that $\Sigma_{1}$ is an invariant region for system (1.1)-(1.2) it is sufficient to prove that the region

$$
\left\{\left(z_{1}^{0}, z_{2}^{0}\right) \in \mathbb{R}^{2}: \quad z_{i}^{0} \geq 0, \quad i=1,2\right\}=[0,+\infty)^{2}
$$

is invariant for system (2.1)-(2.2).

Since, from $(\mathbf{A} 2)$, we have $F_{1}\left(0, z_{2}\right)=0$ for all $z_{2} \geq 0$ and $F_{2}\left(z_{1}, z_{2}\right) \geq 0$ for all $\left(z_{1}, z_{2}\right) \in[0,+\infty)^{2}$, then we obtain $z_{i}(t, x) \geq 0, i=1,2$ for all $(t, x) \in\left[0, T^{*}\right) \times \Omega$, thanks to the invariant regions method (see [25]). As a consequence, $\Sigma_{1}$ is an invariant region for (1.1)-(1.2).

For the case $\Sigma=\Sigma_{2}$, the same reasoning with

- $z_{1}=-\mu_{1} u+v$ and $z_{2}=-\mu_{2} u+v$ for all $(u, v) \in \Sigma_{2}$,

- $F_{1}=-\mu_{1} f+g$ and $F_{2}=\mu_{2} f-g$ for all $(u, v) \in \Sigma_{2}$,

implies the invariance of $[0,+\infty)^{2}$, and then of $\Sigma_{2}$.

\section{Global existence}

Since $\lambda_{1}$ and $\lambda_{2}$ are the eigenvalues of the matrix $A^{t}$, then to prove global existence of solutions for problem (1.1)-(1.4) we need to prove it for problem (2.1)-(2.4).

Since we can use the same way to treat the two cases relating to $\Sigma=\Sigma_{1}$ or $\Sigma=\Sigma_{2}$, we only deal with the first case.

Since, from (A2), we have $F_{1} \geq 0$, then $z_{1}$ satisfies the maximum principle, i.e.,

$$
\left\|z_{1}(t)\right\|_{\infty} \leq\left\|z_{1}^{0}\right\|_{\infty} \quad \text { for all } t \in\left[0, T^{*}\right)
$$

Based on that, the problem of global existence reduces to establish the uniform boundedness of $z_{2}$ in $\left[0, T^{*}\right)$. By $L^{p}$-regularity theory for parabolic operator (see, e.g., [19]) it follows that it is sufficient to derive a uniform estimate of $\left\|F_{2}\left(z_{1}, z_{2}\right)\right\|_{p}$ on $\left[0, T^{*}\right)$ for some $p>\frac{n}{2}$.

The main result is stated in the following key proposition.

Proposition 3.1. Suppose that the assumptions $(\boldsymbol{A} \mathbf{1})-(\boldsymbol{A} \mathbf{4})$ and the restriction (1.6) are fulfilled. For every classical solution $\left(z_{1}, z_{2}\right)$ of (2.1)-(2.4) on $\left[0, T^{*}\right) \times \Omega$, let the function

$$
L: t \longmapsto \int_{\Omega}\left[\delta z_{1}+\left(M-z_{1}\right)^{-\gamma} e^{\alpha p\left(z_{2}+1\right)^{\beta}}\right](x, t) d x,
$$


where $\alpha, \beta, \gamma, \delta, p$ and $M$ are positive constants such that

$$
\beta \geq 1,\left\|z_{1}^{0}\right\|_{\infty}<M<\frac{2 \gamma}{\alpha \beta \ell n} \text { and } \gamma=\frac{4 \lambda_{1} \lambda_{2}}{\left(\lambda_{1}-\lambda_{2}\right)^{2}} .
$$

Then there exists $\delta>0$ and $p>\frac{n}{2}$ such that

$$
L \text { is nonincreasing on }\left[0, T^{*}\right) \text {. }
$$

Before proving this proposition we first need the following lemma.

Lemma 3.2. Let $\left(z_{1}, z_{2}\right)$ be a solution of $(2.1)-(2.4)$ on $\left[0, T^{*}\right) \times \Omega$, then under the assumptions $(\boldsymbol{A} \mathbf{1})-(\boldsymbol{A} 4)$, we have

$$
\int_{\Omega} F_{1}\left(z_{1}(x, t), z_{2}(x, t)\right) d x \leq-\frac{d}{d t} \int_{\Omega} z_{1}(x, t) d x
$$

and there exists $\delta_{1}>0$ and $p>\frac{n}{2}$ such that

$\int_{\Omega}\left[\alpha p \beta M\left(z_{2}+1\right)^{\beta-1} F_{2}\left(z_{1}, z_{2}\right)-\gamma F_{1}\left(z_{1}, z_{2}\right)\right] e^{\alpha p\left(z_{2}+1\right)^{\beta}} d x \leq \delta_{1} \int_{\Omega} F_{1}\left(z_{1}, z_{2}\right) d x$,

where $\alpha, \beta, \gamma$ and $M$ are positive constants satisfying (3.1).

Proof of Lemma 3.2. It suffices to integrate the both sides of (2.1) satisfied by $z_{1}$ on $\Omega$, to obtain (3.3).

Now, from (3.1), we get $\frac{n}{2}<\frac{\gamma}{\alpha \beta \ell M}$, so we can choose $p$ such that $\frac{n}{2}<p<\frac{\gamma}{\alpha \beta \ell M}$. According to the assumption (A3), we have

$$
\begin{aligned}
& {\left[\alpha p \beta M\left(z_{2}+1\right)^{\beta-1} F_{2}\left(z_{1}, z_{2}\right)-\gamma F_{1}\left(z_{1}, z_{2}\right)\right] e^{\alpha p\left(z_{2}+1\right)^{\beta}}} \\
& \leq\left[\alpha p \beta M\left(z_{2}+1\right)^{\beta-1} \psi\left(z_{2}\right)-\gamma\right] e^{\alpha p\left(z_{2}+1\right)^{\beta}} F_{1}\left(z_{1}, z_{2}\right) .
\end{aligned}
$$

Since $\alpha p \beta \ell M<\gamma$ and $(\eta+1)^{\beta-1} \psi(\eta)$ goes to $\ell$ as $\eta \rightarrow+\infty$, there exists $\eta_{0}>0$ such that for all $\eta>\eta_{0}$, we obtain

$$
\left[\alpha p \beta M(\eta+1)^{\beta-1} \psi(\eta)-\gamma\right] e^{\alpha p(\eta+1)^{\beta}} F_{1}(\xi, \eta) \leq 0 .
$$

On the other hand, if $\eta$ is in the compact interval $\left[0, \eta_{0}\right]$, then the continuous function

$$
\eta \longmapsto\left[\alpha p \beta M(\eta+1)^{\beta-1} \psi(\eta)-\gamma\right] e^{\alpha p(\eta+1)^{\beta}}
$$

is bounded. So that (3.4) immediately follows.

Proof of Proposition 3.1. Differentiating $L(t)$ with respect to $t$ and using the Green formula, one obtains

$$
\frac{d}{d t} L(t)=\delta \frac{d}{d t} \int_{\Omega} z_{1}(x, t) d x+I+J
$$


where

$$
\begin{aligned}
& I=\int_{\partial \Omega}\left[\lambda_{1} \gamma \frac{\partial z_{1}}{\partial \nu}+\lambda_{2} \alpha p \beta\left(M-z_{1}\right)\left(z_{2}+1\right)^{\beta-1} \frac{\partial z_{2}}{\partial \nu}\right]\left(M-z_{1}\right)^{-\gamma-1} e^{\alpha p\left(z_{2}+1\right)^{\beta}} d s \\
& -\int_{\Omega}\left[\lambda_{1} \gamma(1+\gamma)\left|\nabla z_{1}\right|^{2}+\alpha p \beta \gamma\left(\lambda_{1}+\lambda_{2}\right)\left(M-z_{1}\right)\left(z_{2}+1\right)^{\beta-1} \nabla z_{1} \nabla z_{2}\right. \\
& \left.+\quad \lambda_{2} \alpha p \beta\left(M-z_{1}\right)^{2}\left(\beta-1+\alpha p \beta\left(z_{2}+1\right)^{\beta}\right)\left(z_{2}+1\right)^{\beta-2}\left|\nabla z_{2}\right|^{2}\right]\left(M-z_{1}\right)^{-\gamma-2} e^{\alpha p\left(z_{2}+1\right)^{\beta}} d x
\end{aligned}
$$

where $d s$ denotes the $(n-1)$-dimensional surface element and

$$
J=\int_{\Omega}\left[\alpha p \beta\left(M-z_{1}\right)\left(z_{2}+1\right)^{\beta-1} F_{2}\left(z_{1}, z_{2}\right)-\gamma F_{1}\left(z_{1}, z_{2}\right)\right]\left(M-z_{1}\right)^{-\gamma-1} e^{\alpha p\left(z_{2}+1\right)^{\beta}} d x .
$$

We now take advantage of (2.4) and $\beta \geq 1$, to obtain that

$$
I \leq-\int_{\Omega} Q\left(\nabla z_{1}, \nabla z_{2}\right)\left(M-z_{1}\right)^{-\gamma-2} e^{\alpha p\left(z_{2}+1\right)^{\beta}} d x
$$

where

$$
\begin{aligned}
Q\left(\nabla z_{1}, \nabla z_{2}\right)= & \lambda_{1} \gamma(1+\gamma)\left|\nabla z_{1}\right|^{2}+\alpha p \beta \gamma\left(\lambda_{1}+\lambda_{2}\right)\left(M-z_{1}\right)\left(z_{2}+1\right)^{\beta-1} \nabla z_{1} \nabla z_{2} \\
& +\lambda_{2}\left(\alpha p \beta\left(M-z_{1}\right)\left(z_{2}+1\right)^{\beta-1}\right)^{2}\left|\nabla z_{2}\right|^{2}
\end{aligned}
$$

is a quadratic form with respect to $\nabla z_{1}$ and $\nabla z_{2}$. The discriminant of $Q$ is given by

$$
D=\gamma\left(\alpha p \beta\left(M-z_{1}\right)\left(z_{2}+1\right)^{\beta-1}\right)^{2}\left[\gamma\left(\lambda_{1}-\lambda_{2}\right)^{2}-4 \lambda_{1} \lambda_{2}\right] .
$$

From conditions (3.1) we have $Q\left(\nabla z_{1}, \nabla z_{2}\right) \geq 0$ and consequently

$$
I \leq 0
$$

Concerning the term $J$, since $0 \leq z_{1} \leq\left\|z_{1}^{0}\right\|_{\infty}<M$, we observe that $J \leq\left(M-\left\|z_{1}^{0}\right\|_{\infty}\right)^{-\gamma-1} \int_{\Omega}\left[\alpha p \beta M\left(z_{2}+1\right)^{\beta-1} F_{2}\left(z_{1}, z_{2}\right)-\gamma F_{1}\left(z_{1}, z_{2}\right)\right] e^{\alpha p\left(z_{2}+1\right)^{\beta}} d x$.

Thanks to (3.4), we get $\delta_{1}>0$ such that

$$
J \leq \delta_{1}\left(M-\left\|z_{1}^{0}\right\|_{\infty}\right)^{-\gamma-1} \int_{\Omega} F_{1}\left(z_{1}, z_{2}\right) d x .
$$

Let $\delta=\delta_{1}\left(M-\left\|z_{1}^{0}\right\|_{\infty}\right)^{-\gamma-1}$ and using (3.3), we obtain

$$
J \leq-\delta \frac{d}{d t} \int_{\Omega} z_{1}(x, t) d x
$$

From (3.5)-(3.7), we conclude that

$$
\frac{d}{d t} L(t) \leq 0 .
$$

This concludes the proof of Proposition 3.1. 
We can now establish the main result of this article.

Theorem 3.3. Under the assumptions (A1)-(A4), the classical solutions of (1.1)-(1.4) with initial data in $\Sigma_{1}$ satisfying (1.6) are global and uniformly bounded on $[0,+\infty) \times \Omega$.

Proof of Theorem 3.3. Let $p$ be the same as in Proposition 3.1. Since $M^{-\gamma} \leq(M-\xi)^{-\gamma}$ for all $\xi \in\left[0,\left\|z_{1}^{0}\right\|_{\infty}\right]$, it follows that

$$
\left\|F_{2}\left(z_{1}, z_{2}\right)\right\|_{p}^{p}=\int_{\Omega}\left|F_{2}\left(z_{1}, z_{2}\right)\right|^{p} d x \leq M^{\gamma} K^{p} L(t)
$$

where

$$
K=\max _{0 \leq \xi \leq\left\|z_{1}^{0}\right\|_{\infty}} \varphi(\xi)
$$

By Proposition 3.1, we deduce

$$
\left\|F_{2}\left(z_{1}, z_{2}\right)\right\|_{p}^{p} \leq M^{\gamma} K^{p} L(0) .
$$

Consequently, $F_{2}\left(z_{1}(t,),. z_{2}(t,).\right)$ is uniformly bounded in $L^{p}(\Omega)$ for all $t \in\left[0, T^{*}\right)$ with $p>\frac{n}{2}$. Using the regularity results for solutions of parabolic equations in [19], we conclude that the solutions of the problem (1.1)-(1.4) are uniformly bounded on $[0,+\infty) \times \Omega$.

Remark 3.4. When $\ell$ is a nonnegative constant, we can replace the restriction (1.6) by

$$
\ell\left\|\mu_{i} u_{0}-v_{0}\right\|_{\infty}<\frac{8 \lambda_{1} \lambda_{2}}{\alpha \beta n\left(\lambda_{1}-\lambda_{2}\right)^{2}} \quad \text { when } \quad \Sigma=\Sigma_{i}, i=1,2,
$$

and we observe that if $\ell=0$, then the initial conditions in $\Sigma$ are given arbitrarily.

Acknowledgments. The first author would like to thank the kind hospitality of the Elie Cartan Institute of Nancy, where this work was done.

\section{REFERENCES}

[1] N. D. Alikakos, $L^{p}$-bounds of solutions of reaction-diffusion equations, Comm. Partial Differential Equations 4 (1979), 827-868.

[2] H. Amann, Dynamic theory of quasilinear parabolic equations - I. Abstract evolution equations, Nonlinear Anal. 12 (1988), 895-919.

[3] A. Barabanova, On the global existence of solutions of a reaction-diffusion equation with exponential nonlinearity, Proc. Amer. Math. Soc. 122 (1994), $827-831$.

[4] N. Boudiba and M. Pierre, Global existence for Coupled Reaction-Diffusion Systems, J. Math. Ana. and Appl. 250 (2000), pp. 1-12.

[5] E. L. Cussler, Multicomponent diffusion, Chemical Engineering Monographs, Vol. 3, Elsevier Publishing Scientific Company, Amesterdam, 1976. 
[6] E. L. Cussler, Diffusion, Mass Transfer in Fluid Systems, Second Edition, Cambridge University Press, 1997.

[7] A. Friedman, "Partial differential equations of parabolic type", Prentice Hall Englewood Chiffs. N. J. 1964.

[8] A. Haraux and A. Youkana, On a result of $K$. Masuda concerning reactiondiffusion equations, Thoku Math. J. 40 (1988), 159-163.

[9] D. Henry, "Geometric theory of semilinear parabolic equations", Lecture Notes in Mathematics 840, Springer-Verlag, New York, 1981.

[10] M. A. Herrero, A. A. Lacey and J. J. L. Velazquez, Global existence for reaction-diffusion systems modelling ignition, Arch. Rational Mech. Anal. 142 (1998), 219-251.

[11] S. L. Hollis, R. H. Martin and M. Pierre, Global existence and boundedness in reaction-diffusion systems, SIAM J. Math. Anal. 18 (1987), 744-761.

[12] J. I. Kanel and M. Kirane, Pointwise a priori bounds for a strongly coupled system of reaction-diffusion equations with a balance law, Math. Methods Appl. Sci. 21 (1998), 1227-1232.

[13] J. I. Kanel and M. Kirane, and N. E. Tatar, Pointwise a priori bounds for a strongly coupled system of reaction-diffusion equations, Int. J. Differ. Equ. Appl. 1 (2000), 77-97.

[14] M. Kirane and S. Kouachi, Global solutions to a system of strongly coupled reaction-diffusion equations, Nonlinear Anal. 26 (1996), 1387-1396.

[15] S. Kouachi, Global existence of solutions for reaction-diffusion systems with a full matrix of diffusion coefficients and nonhomogeneous boundary conditions, Electron. J. Qual. Theory Differ. Equ. 4 (2002), pp. 1-10.

[16] S. Kouachi, Global existence of solutions in invariant regions for reactiondiffusion systems with a balance law and a full matrix of diffusion coefficients, Electron. J. Qual. Theory Differ. Equ. 2 (2003), pp. 1-10.

[17] S. Kouachi, Invariant regions and global existence of solutions for reactiondiffusion systems with full matrix of diffusion coefficients and nonhomogeneous boundary conditions, Georgian Math. J. 11 (2004), 349-359.

[18] S. Kouachi and A. Youkana, Global existence for a class of reaction-diffusion systems, Bull. Polish Acad. Sci. Math. 49 (2001), 303-308.

[19] O. A. Ladyzenskaja, V. A. Solonnikov and N. N. Uralceva, "Linear and quasilinear equations of parabolic type", Amer. Math. Soc. 1968.

[20] D. Lamberton, Equations d'évolution linéaires associées a des semi-groupes de contractions dans les espaces $L^{p}$, J. Funct. Anal. 72 (1987), 252-262.

[21] K. Masuda, On the global existence and asymptotic behavior of solutions of reaction-diffusion equations, Hokkaido Math. J. 12 (1983), 360-370.

[22] A. Pazy, "Semigroups of linear operators and applications to partial differential equations", Appl. Math. Sci. 44, Springer-Verlag, New York 1983.

[23] M. Pierre and D. Schmitt, Blow up in reaction-diffusion systems with dissipation of mass, SIAM J. Math. Anal. 28 (1997), 259-269.

[24] B. Rebiai and S. Benachour, Global classical solutions for reaction-diffusion systems with nonlinearities of exponential growth, (in press).

[25] J. Smoller, "Shock waves and reaction-diffusion equations", Springer-Verlag, New York 1983. 
Belgacem Rebiai: Département de Mathématiques, Université de TÉBessa, 12002, Algérie.

Et Institut Elie Cartan, Université Henri Poincaré, F-54506 VandeuvreLès-Nancy Cedex, France.

E-mail address: Rebiai.Belgacem@iecn.u-nancy.fr

Saïd Benachour: Institut Elie Cartan, Université Henri Poincaré, F-54506 VAndeuvre-Lès-NanCy Cedex, France.

E-mail address: Said.Benachour@iecn.u-nancy.fr 